\title{
BMJ Open Uptake of voluntary medical male circumcision among men with sexually transmitted infections in Lilongwe, Malawi: a protocol for a pre- interventional and post- interventional study
}

\author{
Mitch M Matoga (D) , ,2 Mina C Hosseinipour, ${ }^{1,3}$ Sara Jewett (D) ,4 \\ Charles Chasela ${ }^{2,5}$
}

To cite: Matoga MM, Hosseinipour MC, Jewett S, et al. Uptake of voluntary medical male circumcision among men with sexually transmitted infections in Lilongwe, Malawi: a protocol for a pre-interventional and postinterventional study. BMJ Open 2022;12:e057507. doi:10.1136/ bmjopen-2021-057507

- Prepublication history and additional supplemental material for this paper are available online. To view these files, please visit the journal online (http://dx.doi.org/10.1136/ bmjopen-2021-057507)

Received 20 September 2021 Accepted 04 January 2022

Check for updates

(C) Author(s) (or their employer(s)) 2022. Re-use permitted under CC BY-NC. No commercial re-use. See rights and permissions. Published by BMJ.

For numbered affiliations see end of article.

Correspondence to Dr Mitch M Matoga; mmatoga@unclilongwe.org

\section{ABSTRACT}

Introduction Voluntary medical male circumcision (VMMC) is one of the key interventions for HIV prevention. However, its uptake among men in Malawi is low. Implementation science strategies for demand creation of VMMC increase uptake. We designed an implementation science demand-creation intervention to increase the uptake of VMMC among men with sexually transmitted infections (STIs).

Methods and analysis We designed a pragmatic preinterventional and post-interventional quasi-experimental study combined with a prospective observational design to determine the uptake, acceptability, appropriateness and feasibility of a multifaceted intervention for scale up of uptake of VMMC among men with STIs at Bwaila STI clinic in Lilongwe, Malawi. The intervention includes transport reimbursement $(\mathrm{R})$, intensified health education (I) and short messaging service (SMS)/telephonic tracing (Te) (RITe). The intervention will be implemented in phases: pre-implementation and implementation. Pre-implementation phase will be used for collecting baseline data, while the RITe intervention will be rolledout in the implementation phase. The RITe intervention will be implemented in a sequential and incremental manner called implementation blocks: block 1 : intensified health education; block 2: intensified health education and SMS/telephonic tracing; and block 3: intensified health education, SMS/telephonic tracing and transport reimbursement. The target sample size is 80 uncircumcised men for each intervention block, including the pre-implementation sample, making a total of 320 men ( 280 total, 70 per block will be surveyed). The primary outcome is uptake of VMMC during the implementation period. Mixed methods assessments will be conducted to evaluate the acceptability, appropriateness and feasibility of the RITe intervention.

Ethics and dissemination The study protocol was approved by the Malawi's National Health Sciences Research Ethics Committee (approval number: 19/10/2412), University of North Carolina at Chapel Hill's Institutional Review Board (approval number: 19-2559)
Strengths and limitations of this study

- We designed an implementation science demandcreation intervention (transport reimbursement (R), intensified health education (I) and short messaging service/telephonic tracing (Te) (RITe)) that can be adopted or adapted in other settings to increase uptake of voluntary medical male circumcision (VMMC) among men at high risk of HIV acquisition.

- Our study will use a pre-intervention and postintervention quasi-experimental design combined with a prospective observational study design and mixed methods approach to evaluate the intervention's impact on uptake of VMMC, and the intervention's acceptability, appropriateness and feasibility.

- As a limitation, our study will not capture men who undergo circumcision after 30 days of follow-up, which may lead to an underestimation of the impact of the intervention if some men will circumcise after the follow-up period.

- Another limitation is that participants will not be randomised into intervention groups, thus may introduce some bias; however, the pragmatic study design will evaluate the impact of the intervention in a 'real-world' setting.

and University of the Witwatersrand's Health Research Ethics Committee (approval number: M200328). Results will be disseminated via publication in a peer-reviewed journal and presentations at relevant scientific conferences and meetings.

Trial registration number NCT04677374.

\section{INTRODUCTION}

Voluntary medical male circumcision (VMMC) is one of the essential interventions earmarked by the Joint United Nations Programme on HIV/AIDS (UNAIDS) for ending AIDS as a public health threat by 
2030. ${ }^{1}$ Since 2007 , high-priority countries in eastern and southern Africa recommended by the WHO and UNAIDS due to high HIV and low male circumcision prevalence have scaled up VMMC activities. ${ }^{2}$ In 2016, the UNAIDS set a global VMMC Fast-Track target recommending an additional 25 million boys and men in high-priority countries to receive VMMC by $2020 .{ }^{1}$ As a result, about 15 million VMMCs were conducted between 2016 and $2020 .{ }^{3}$ Despite the remarkable progress in the last few years, the cumulative performance fell $40 \%$ short of the 25 million target. $^{3}$

Malawi, a VMMC high-priority country, adopted VMMC as an HIV prevention strategy in 2012. ${ }^{4}$ At the inception of the national VMMC programme, the target was to circumcise $60 \%$ of men aged $10-34$ years by $2020 .{ }^{4}$ In 2016 , the Fast-Track target for Malawi was to achieve 2458727 VMMCs across the country by $2020 .{ }^{4}$ However, only about 939573 VMMCs were conducted by 2020, approximately $38 \%$ of the target. ${ }^{3}$ Religion and culture are the main determinants of uptake of VMMC in Malawi and they influence the geographical distribution of male circumcision. ${ }^{5}$ Other reasons include fear of infection, bleeding and pain, costs associated with accessing VMMC and limited access to VMMC services, among other factors. ${ }^{6-11}$ To achieve high impact, Malawi selected 12 high-priority districts based on low male circumcision and high HIV incidence rates. The current national VMMC target is to circumcise $80 \%$ of men aged 15-49 years (about 940000 ) in high-priority districts by 2025 and to maintain current coverage (around $30 \%$ ) in low-priority districts. ${ }^{12}$

In order to achieve this ambitious target, effective implementation strategies are required. The UNAIDS and WHO recommend prioritising adolescents and young men (10-29 years) for a greater public health benefit. ${ }^{13}$ But, recent literature shows that including older and higher-risk men would amplify impact in a shorter timeframe. ${ }^{1415}$ An important population of highrisk men to prioritise for VMMC is men with sexually transmitted infections (STIs) ${ }^{15}$ as STIs increase the risk of HIV acquisition by at least threefold. ${ }^{16}$ Since VMMC reduces the risk of female-to-male HIV transmission by $60 \%{ }^{17-19}$ and also protects against several STIs, ${ }^{20} \mathrm{VMMC}$ is an ideal intervention for STI clinics and STI clinics are an ideal setting for VMMC demand creation. Recently, integration of VMMC recruitment in STI clinics was shown to be an effective VMMC demand creation strategy in Malawi. ${ }^{15}$ However, the uptake of VMMC was only $10 \%$, underscoring the need for innovative efforts to increase demand among men with STIs. ${ }^{15}$

To address the low uptake of VMMC among men with STIs, we designed a multifaceted demand creation intervention consisting of use of transport reimbursement (R), intensified health education (I) and short messaging service (SMS)/telephonic tracing (Te) (the RITe intervention). Our aim is to estimate the effectiveness of the RITe intervention in increasing the uptake of VMMC among men with STIs at Bwaila STI clinic in Lilongwe, Malawi. The study's specific objectives are (1) to compare uptake and time to uptake of VMMC between the RITe intervention and the standard of care, (2) to assess factors associated with uptake of VMMC among the men and (3) to assess the acceptability, appropriateness and feasibility of the RITe intervention among men and healthcare workers (HCWs). We hypothesise that the RITe intervention will effectively increase the uptake of VMMC to $60 \%$ (the overall national target), that the intervention will be viewed as acceptable and appropriate by men and HCWs and that implementation of the intervention will be feasible at Bwaila STI clinic.

\section{METHODS AND ANALYSIS Study overview}

This is a pragmatic, pre-interventional and postinterventional quasi-experimental study combined with a prospective observational study design. The study will have pre-implementation and implementation phases. The pre-implementation phase (2-4 months) will be used to collect baseline data on uptake of VMMC during the standard of care period, and to assess acceptability and appropriateness of the RITe intervention at baseline. The intervention will be rolled-out in the implementation phase in a sequential and incremental manner called implementation blocks. Implementation blocks will be as follows: block 1: intensified health education; block 2: intensified health education and SMS/telephonic tracing and finally, block 3: intensified health education, SMS/telephonic tracing and transport reimbursement (figure 1). Each participant within the implementation blocks will be followed for 30 days to observe if they will undergo circumcision. A concurrent exploratory mixed methods approach will be used to evaluate the acceptability, appropriateness and feasibility of each implementation block. Noteworthy, the intervention was initially designed to include clinic escort to the VMMC clinic co-located within the Bwaila hospital. However, clinic escort will be excluded because the VMMC clinic has been repurposed to a COVID-19 isolation unit. All men interested in circumcision will be linked to the nearest clinic of their choice offering VMMC services.

Based on sample size estimates, each implementation block is expected to last approximately 1-3 months. There will be a 1-week break (the washout period) between implementation blocks to avoid overlap of the effect of the intervention strategies (figure 1). This study approach will allow us to compare the effectiveness of different combinations of the RITe intervention to the standard of care without necessarily randomising the intervention. Details of the interventions are provided under study intervention.

\section{Study setting}

The study will be conducted at the Bwaila District Hospital $(\mathrm{BDH}) . \mathrm{BDH}$ is a public secondary level facility with a catchment area of about 1.4 million people and the only 

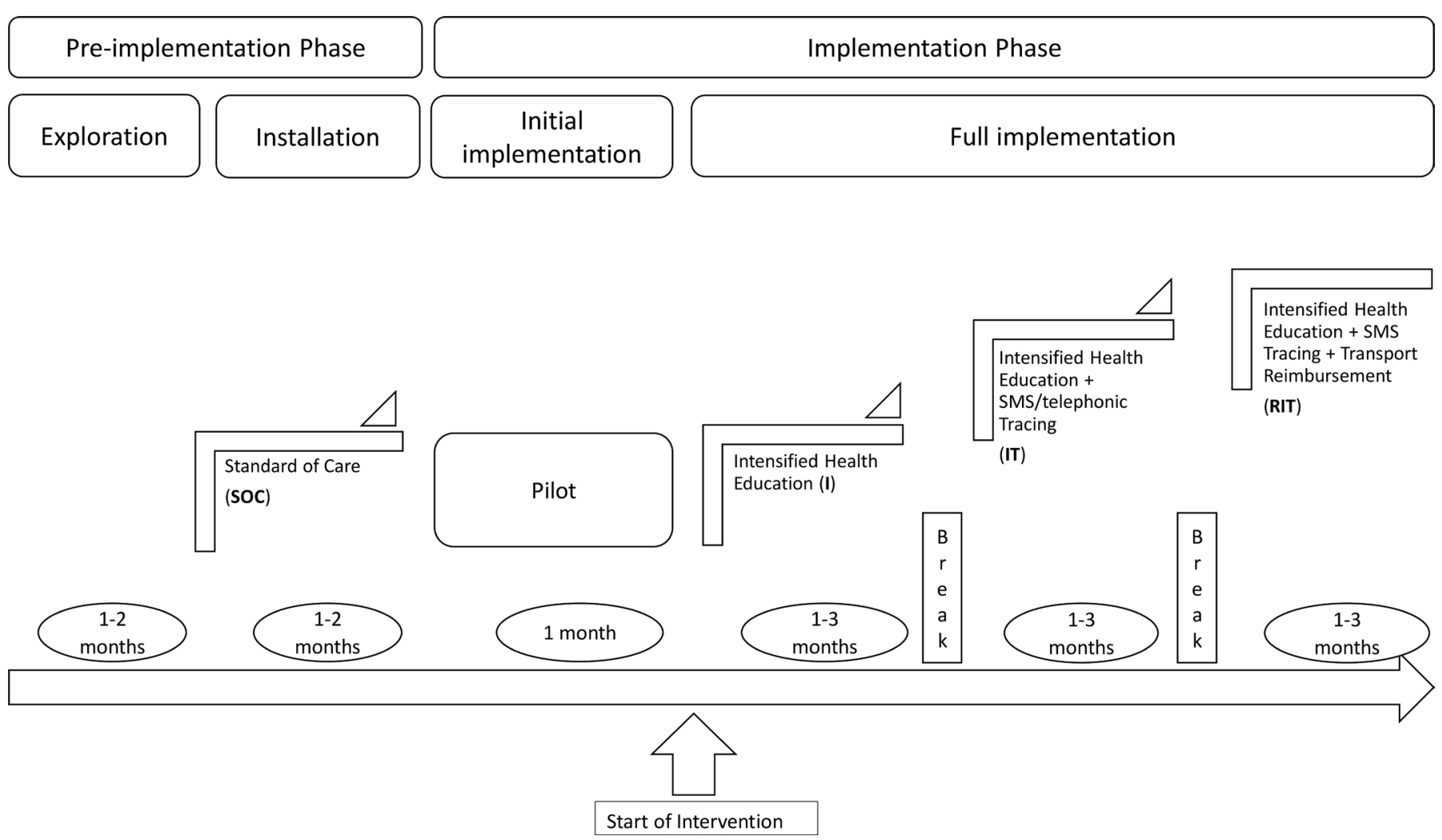

Figure 1 Overview of study phases, stages and intervention roll-out.

facility offering specialised STI care situated in a busy central town area of the Lilongwe district.

The Bwaila STI clinic has approximately 13000 patient visits per year and is staffed by nurses, clinicians, HIV testing services counsellors and other support staff. As standard practice, all patients receive group health education on HIV and STIs and are offered routine opt-out HIV testing, before receiving care for their STI. Education on VMMC has not been routinely conducted since late 2020 due to completion of a US President's Emergency Plan for AIDS Relief (PEPFAR) funded project. Health education is conducted by the clinic receptionist, rarely assisted by a counsellor. Offering VMMC to all uncircumcised men receiving STI care is part of policy in Malawi but not standard practice at Bwaila STI clinic.

VMMC services are offered freely by the Ministry of Health. The Ministry of Health receives programme support largely through implementing partners in PEPFAR supported districts. There are 12 priority districts in Malawi, including Lilongwe. Since the closure of the VMMC clinic at BDH, men interested in VMMC are referred to free public primary health facilities around the district. Selected public facilities have trained VMMC providers who conduct medical circumcision as an outpatient service. As of 2017, the national circumcision prevalence rate among males aged 15-49 years was $28 \%, 18.0 \%$ circumcised by traditional practitioners and $9.0 \%$ by medical professionals. ${ }^{21}$ Among patients with STI, the prevalence of circumcision in 2018 was also estimated at $28 \% .^{22}$ At Bwaila STI clinic, an unpublished report showed a circumcision prevalence of $19.3 \%$ in 2017.

The overall HIV prevalence among adults aged 15-64 years in Malawi is $10.6 \%$, and $11.5 \%$ in the Lilongwe city. ${ }^{23}$ The HIV prevalence among patients with STI in Malawi is around $18.0 \%-22.0 \%{ }^{152}$ and around $13.3 \%$ at Bwaila STI clinic. ${ }^{25}$

\section{Study population and eligibility}

The main study population is comprised of (1) uncircumcised men, (2) aged $\geq 18$ years and (3) accessing STI care regardless of their HIV status, at Bwaila STI clinic during the study period. Men who are already circumcised will not participate. The study population will also include HCWs at Bwaila STI and other key stakeholders (VMMC providers, programme implementers and policy-makers).

\section{Study intervention: conceptual framework, intensified health education, SMS/telephonic tracing and transport reimbursement \\ Conceptual framework}

The RITe intervention was developed based on literature and expert consultation. The intervention design was guided by a conceptual framework (figure 2) and the refined compilation of implementation strategies from the Expert Recommendations for Implementing Change project. ${ }^{26}$ The conceptual framework was developed by adapting the framework for demand generation, ${ }^{27}$ the model for promotion of uptake of male circumcision ${ }^{28}$ and the National Implementation Research Network 


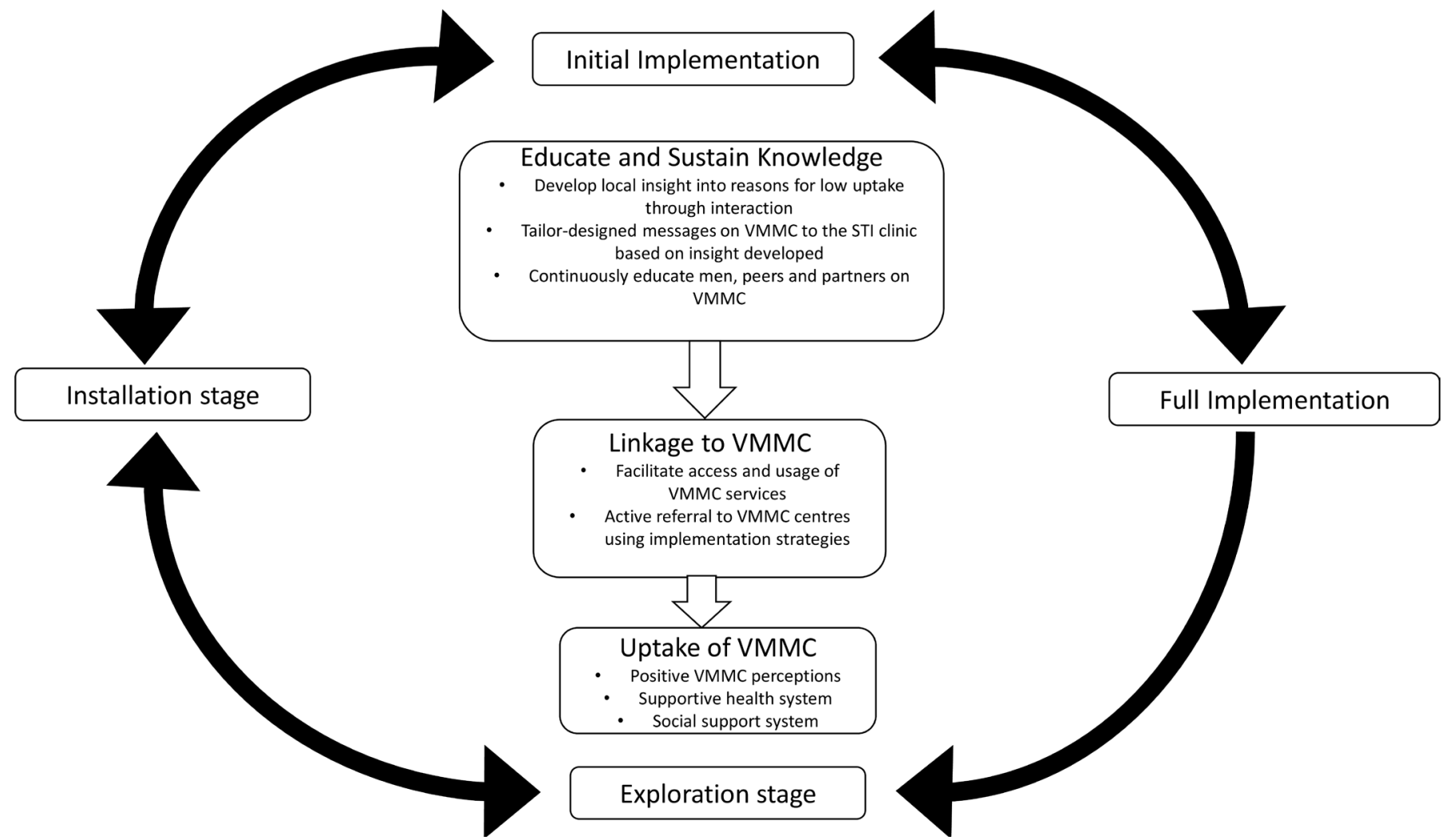

Figure 2 Conceptual framework for increasing uptake of VMMC. STI, sexually transmitted infection; VMMC, voluntary medical male circumcision.

(NIRN) stages of implementation. ${ }^{29}$ The framework anchors on the theory that knowledge is an integral component of perceptions and that perceptions are a major determinant of behavioural change. Therefore, the rationale of the framework is to provide men with adequate and accurate knowledge about VMMC in order to improve their perceptions about VMMC, and linking them to the nearest VMMC clinic to ensure access, and, therefore, increasing their demand for VMMC.

To provide knowledge, we will first develop insight on the factors that influence decision-making on VMMC among men with STIs at the Bwaila STI clinic and from VMMC experts/stakeholders, then develop health education content based on the insight (educate and sustain step of the conceptual framework), guided by the Malawi National VMMC Communication Strategy. ${ }^{4}$ We hypothesise that the education will improve perceptions about VMMC among men attending the clinic, including their partners and peers, and will help to nullify misconceptions about VMMC. Under 'linkage and access to care' in the conceptual framework, we aim to facilitate access and utilisation of VMMC services for interested men by actively referring men to their clinic of choice, reminding them of their VMMC appointments and supporting them by reimbursing the cost of transportation. In turn, we anticipate that the men who will receive VMMC will influence other men in the community to also undergo VMMC (figure 2).
Intensified health education (I)

Standard of care group health education is usually conducted once or twice a day at Bwaila STI clinic. As stated earlier, health education on VMMC was discontinued late 2020. Before discontinuation, the content of the VMMC education focused on benefits of VMMC. In line with the Malawi VMMC communication strategy, we will develop a health education guide through expert consultation (stakeholder survey), and interviews with men and HCWs at the STI clinic. The education is expected to contain more detailed explanation of VMMC tailored to men with STIs. In addition to benefits of VMMC, we will seek consensus from the men and experts on the value of including content on risks associated with VMMC and dispelling myths and misconceptions about VMMC during health talks. Education on VMMC will be offered through more regular and frequent (at least four times per day) health education sessions by a trained VMMC mobiliser and VMMC champions (a man who has received circumcision and a woman whose partner has received circumcision). Furthermore, men interested in VMMC will be offered one-on-one counselling with the mobiliser and champions. Similarly, women willing to know more about circumcision will also be offered oneon-one counselling. Comprehensive group education and one-on-one counselling are effective strategies for increasing the uptake of VMMC. ${ }^{30} 31$ The combination of comprehensive and STI setting-specific group education 
with one-one-one counselling will form the intensified health education. Further justification for the intensified health education strategy has been included in the Discussion section.

\section{SMS/telephonic tracing (Te)}

We will use SMSs and/or phone calls to remind men of their appointments for circumcision. Men will receive an SMS on day 1, day 3 and day 7 after their STI clinic visit. Phone calls will be attempted if patients will not receive circumcision by day 14 . The SMS will consist of a reminder to receive circumcision at the nearest clinic of choice. The SMS will not contain any patient identifiable information. A recent systematic review showed benefits of SMS reminders in improving many clinical outcomes, ${ }^{32}$ including improved VMMC postoperative visits. ${ }^{33}$ Further justification for this strategy has been detailed in the Discussion section.

\section{Transport reimbursement ( $\mathrm{R})$}

We propose to compensate the cost associated with circumcision by providing a transport reimbursement of US $\$ 10$ based on the Malawi's National Health Sciences Research Ethics Committee (NHSRC) guidelines. Men will receive the compensation on receipt of circumcision confirmed by the provider through adequate documentation in the health registers and passport books or by contacting the VMMC provider who performed the circumcision. On confirmation of circumcision, reimbursements will be provided in cash at Bwaila STI clinic. Economic compensations of any form, such as food/transport vouchers or conditional cash transfers, have effectively increased uptake of VMMC and are well accepted. ${ }^{34}{ }^{35}$ Justification for transport reimbursement has been detailed in the Discussion section.

\section{Study implementation: pre-implementation and implementation phases}

Pre-implementation phase (exploration and installation stages)

The pre-implementation phase, consisting of the exploration and installation NIRN stages, will be for planning and creating readiness for implementation and collection of baseline data. Exploration will include identification and mobilisation of resources required for implementation. We will identify one VMMC mobiliser and two VMMC champions among satisfied VMMC clients from key stakeholders. Key stakeholders will be briefed on the study to obtain buy-in. The installation stage will be used to prepare study tools and train the research team, including the VMMC mobiliser and champions on the intervention. We will conduct formative interviews with men and HCWs and a survey with experts (stakeholders) to obtain insight on factors that hinder men with STIs from receiving VMMC. The findings will be used to develop content for the intensified health education. The mobiliser and champions will be trained on the content of the intensified health education, interpersonal communication and relevant information about HIV and
STIs. In addition, we will collect baseline data on uptake of VMMC and conduct baseline assessments for acceptability and appropriateness (figure 1).

\section{Implementation phase (initial implementation and full implementation)}

In the implementation phase, under initial implementation, we will pilot the intensified health education script and refine it. Afterwards, we will roll-out our intervention starting with block 1 , then block 2 and finally block 3 (full implementation) (figure 1). There will be a 1-week wash-out period after each implementation block. This study opened to accrual in February 2021 and data collection is currently underway. However, due to the COVID-19 pandemic, there has been a delay in recruitment, with anticipation to complete data collection by June 2022 and to complete the study by December 2022. Duration of data collection for each intervention block will be extended until sample size is reached.

\section{Study procedures: recruitment, baseline and follow-up procedures}

Recruitment for this study will be conducted at Bwaila STI clinic. Men will be educated about circumcision during the group health talks and offered circumcision. Men interested in circumcision will then undergo one-on-one counselling with the VMMC mobiliser and champions. Women willing to refer their partners for recruitment will also undergo one-on-one counselling. Men will receive different intervention packages depending on the intervention block that coincides with their initial visit to the clinic. The process of informed consent for study participation will be fully documented. Only men who will provide written informed consent (online supplemental file 1) will participate in the surveys and focus group discussions (FGDs). As standard of care, men who are willing to undergo circumcision will provide written informed consent for the procedure at their clinic of choice. We will also recruit HCWs from the clinic who will participate in surveys and in-depth interviews (IDIs). HCWs will also provide written informed consent to participate in the study (online supplemental file 1). Experts (key stakeholders) will provide verbal consent to participate in a survey.

\section{Baseline procedures}

For the men who will enrol, we will collect data on demographics, HIV history, sexual history, current STI diagnosis and contact information using the electronic medical registry already in existence at Bwaila STI clinic. Demographic data will include age, marital status, level of education and socioeconomic status. Sexual history will include date of last sexual contact and frequency, and condom use. Contact information will be used to contact men to remind them of their VMMC appointments in the implementation phase. In addition, we will conduct two baseline FGDs and a survey with patients, IDIs and a survey with willing HCWs and a survey with experts. 


\section{Follow-up procedures}

Each participant will be followed for 30 days to determine whether or not they received circumcision. We will collect data on circumcision status by contacting VMMC providers in public facilities and/or by review of documentation in the patients' health passport books. We will conduct end-line IDIs and surveys with HCWs, and another two FGDs and surveys with the patients.

\section{Study measure and outcomes}

Primary outcomes

The primary outcomes for this study are uptake, time to uptake and factors associated with uptake of VMMC. Uptake will be defined as the action of undergoing medical circumcision. We will collect data on the number of uncircumcised men who receive VMMC for each implementation block. Time to uptake of VMMC will be the number of days taken between the date a patient is offered circumcision and the date they will undergo circumcision. The uptake and time to uptake will be compared between the intervention and the standard of care. In addition, using demographic, HIV history, sexual history and current STI diagnosis history data, we will explore factors associated with uptake of VMMC, triangulated with qualitative data (table 1).

\section{Secondary outcomes}

Secondary outcomes will be acceptability, appropriateness and feasibility of the RITe intervention to HCWs and patients. The secondary implementation outcomes will be defined as follows:

1. Acceptability will be the perception among men and HCWs that the RITe intervention is agreeable, palatable and satisfactory. ${ }^{36}$

2. Appropriateness will be defined as the perception among men and HCWs that the RITe intervention is a good fit or relevant. ${ }^{36}$

Table 1 Description of procedures and assessments by objective

Assessment Description Participants Sample size

Objective 1: to compare uptake within 30 days and time to uptake of VMMC between the RITe intervention and the standard of care

Uptake of VMMC

Time to uptake of VMMC

The number of uncircumcised Men aged $\geq 18$ years

men who will undergo VMMC

within 30 days from the date

offered

The number of days from the date offered to date received circumcision

Objective 2: to assess factors associated with uptake of VMMC

\begin{tabular}{|c|c|c|c|}
\hline $\begin{array}{l}\text { Factors associated with uptake } \\
\text { of VMMC }\end{array}$ & $\begin{array}{l}\text { Demographic, sexual, HIV } \\
\text { and clinical factors affecting } \\
\text { uptake of VMMC } \\
\text { IDIs with HCWs } \\
\text { FGDs with men } \\
\text { Expert (stakeholder) survey }\end{array}$ & $\begin{array}{l}\text { Men aged } \geq 18 \text { years } \\
\text { HCWs } \\
\text { Experts }\end{array}$ & $\begin{array}{l}320 \text { men ( } 80 \text { in each } \\
\text { implementation block) } \\
\text { All available HCWs }(\sim 12) \text { and } \\
\text { experts }(\sim 10)\end{array}$ \\
\hline
\end{tabular}

Objective 3: to assess the acceptability, appropriateness and feasibility of the RITe intervention

\begin{tabular}{|c|c|c|c|}
\hline Acceptability & $\begin{array}{l}\text { Assessment will be through } \\
\text { AIM Likert scale survey, IDIs } \\
\text { and FGDs } \\
\text { Assessment will be at baseline } \\
\text { and end line }\end{array}$ & $\begin{array}{l}\text { Men aged } \geq 18 \text { years } \\
\text { (surveys and FGDs) } \\
\text { HCWs (surveys and IDIs) }\end{array}$ & $\begin{array}{l}70 \text { men for the surveys. } 6-10 \\
\text { men for the FGDs } \\
\text { All HCWs at Bwaila STI clinic } \\
(\sim 12)\end{array}$ \\
\hline Appropriateness & $\begin{array}{l}\text { Assessment will be through } \\
\text { IAM Likert scale survey, IDIs } \\
\text { and FGDs } \\
\text { Assessment will be at baseline } \\
\text { and end line }\end{array}$ & $\begin{array}{l}\text { Men aged } \geq 18 \text { years } \\
\text { (surveys and FGDs) } \\
\text { HCWs (surveys and IDIs) }\end{array}$ & $\begin{array}{l}70 \text { men for the surveys. } 6-10 \\
\text { men for the FGDs } \\
\text { All HCWs at Bwaila STI clinic } \\
(\sim 12)\end{array}$ \\
\hline Feasibility & $\begin{array}{l}\text { Assessment will be through } \\
\text { FIM Likert scale survey, IDIs } \\
\text { and FGDs } \\
\text { Assessment will be at end line } \\
\text { only }\end{array}$ & $\begin{array}{l}\text { Men aged } \geq 18 \text { years } \\
\text { (surveys and FGDs) } \\
\text { HCWs (surveys and IDIs) }\end{array}$ & $\begin{array}{l}70 \text { men for the surveys. } 6-10 \\
\text { men for the FGDs } \\
\text { All HCWs at Bwaila STI clinic } \\
(\sim 12)\end{array}$ \\
\hline
\end{tabular}

AIM, acceptability of intervention measure; FGDs, focus group discussions; FIM, feasibility of intervention measure; HCWs, healthcare workers; IAM, intervention appropriateness measure; IDIs, in-depth interviews; RITe, transport reimbursement (R), intensified health education (I) and SMS/telephonic tracing (Te); STI, sexually transmitted infection; VMMC, voluntary medical male circumcision. 
3. Feasibility will be the extent to which RITe intervention will be successfully implemented at Bwaila STI clinic. ${ }^{36}$

All secondary outcomes will be measured using concurrent exploratory mixed methods approach. For quantitative measurements, we will administer Likert scale surveys, while IDIs and FGDs will be used for qualitative assessments. The quantitative and qualitative data will be triangulated. All outcomes will be measured at baseline and end line, except for feasibility, which will only be measured at end line, after exposure to the intervention (table 1).

Psychometric tools: the acceptability of intervention measure (AIM), intervention appropriateness measure (IAM) and feasibility of intervention measure (FIM) will be used for the Likert scale surveys. ${ }^{37}$ Surveys will be conducted for each implementation block and at baseline, except for feasibility, which will be assessed in the implementation phase only. Surveys will be self-administered for HCWs and assisted for men using open data kit.

\section{Qualitative data collection}

Qualitative assessments will be conducted using IDIs for HCWs and FGDs for men at baseline and end line (table 1). IDIs will be used for HCWs, allowing us to obtain individual perspectives with more depth. We want HCWs to be able to respond both from their perspectives as providers as well as individuals, so that considerations like gender can be discussed freely. IDIs will be conducted with all consenting HCWs at Bwaila STI clinic.

FGDs will be conducted with men, instead of IDIs, as we want to draw out the social and peer dynamics of VMMC decision-making in the context of RITe. Each FGD will be conducted among a subset of 6-10 men. Two FGDs will be conducted at baseline and another two at end line. The investigator developed semi-structured IDI and FGD guides using the AIM, IAM and FIM tools. The guides were translated into Chichewa by two translators at the University of North Carolina Project and the IDIs and FGDs will be conducted in Chichewa. All IDIs and FGDs will be conducted by the investigator and a qualitative researcher. Demographic data for respondents will be collected on a separate form. The IDIs and FGDs will be audio recorded with consent from the respondents and supplemented with field notes taken during and after the interviews or discussions (table 1). Audio recordings will be transferred into an encrypted computer with passwords only known to the transcriber, then recordings in the recorder will be deleted. Audio recordings will be transcribed and translated directly into English by the investigator and a qualitative researcher.

\section{Statistical analysis, power and sample size estimation Sample size estimation}

As indicated earlier, only 28\% of Malawian men with STIs were circumcised by 2018. Sample size calculation was estimated to increase the prevalence of medical circumcision among men with STIs from $28 \%$ to $60 \%$ (32\% difference), the national target at study conceptualisation. We assumed that the population exposed to our intervention will be different with each implementation block. Using a two-sided precision rate of $0.05 \%$ and $80 \%$ power, we estimated a minimum sample size of 55 uncircumcised men for each intervention block. Based on observations from previous studies, we anticipate a refusal rate of $25 \%$. To cater for the refusal rate, we estimated the sample size to 69 men. Furthermore, among the enrolled men, we anticipate a 1-month follow-up drop-out rate of around $15 \%$. To cater for the drop-out rate, we estimated the sample size to 80 uncircumcised men for each intervention block. Including the standard of care, the estimated total sample size will be approximately 320 uncircumcised men.

Based on the sample size of 80 men per block, at $95 \%$ confidence level and 5\% margin of error, we estimated a sample size of 67 men per block to participate in the surveys using a survey sample size calculator (https:// www.surveymonkey.com/mp/sample-size-calculator/). However, based on previous surveys conducted at Bwaila STI clinic, we anticipated a $5 \%$ non-response rate; therefore, the sample size was re-estimated to 70 men per block. All (approximately 12) HCWs will be offered to take the surveys. All available experts (approximately 10) will be offered to take a survey.

We will use descriptive statistics such as mean, median and proportion to describe the demographic characteristics of the study population. To compare uptake between the standard of care and the RITe intervention, uptake of circumcision will be calculated as a proportion of men who will undergo VMMC against the number of men offered VMMC. We will calculate the uptake and the corresponding 95\% CI for each implementation block and for the entire implementation phase, and compare with the standard of care using two-sample test of proportions test at $\alpha=0.05$ significance level. To measure time to uptake of circumcision, data analysis will be restricted to the period of follow-up (30 days). Time to uptake of circumcision for each implementation block and for the overall intervention will be analysed using Kaplan-Meier methods and compare with standard of care using the logrank test. Unadjusted and adjusted proportional hazards models will be used to evaluate factors associated with uptake of VMMC and report HRs with corresponding 95\% CIs. Factors associated with uptake of VMMC will also be explored qualitatively using IDIs and FGDs as detailed below.

For the Likert scale surveys, using the AIM, IAM and FIM, overall median scores and IQRs for acceptability, appropriateness and feasibility will be calculated and compared the baseline and end-line acceptability and appropriateness scores using the Wilcoxon rank sum test. The scores will range from 6 to 80 for AIM, from 5 to 85 for IAM and from 5 to 60 for FIM. The overall median scores will be expressed as percentages. We will calculate percentages for each construct of acceptability, appropriateness and feasibility and plot on bar charts to display the percentage who strongly agree, agree, neutral, disagree or strongly disagree. 
For qualitative data, thematic analysis will be facilitated by NVivo V.12 Pro 2018. The investigator and a qualitative researcher will develop a codebook that will be used to categorise and reduce the raw data. Initial codes will originate from the main questions on the guides and additional codes will be added using both inductive and deductive approaches. Coding will be conducted by the investigator and a qualitative researcher. Codes will be compared for intercoder reliability. In cases of coding inconsistencies, the investigator and a qualitative researcher will discuss and agree on a code. Once all transcripts are coded, the investigators will group codes into common themes. Patterns between and within themes will be explored to better understand factors influencing VMMC uptake among men with STIs as well as the acceptability, appropriateness and feasibility of the RITe intervention.

\section{Patient and public involvement}

The public was engaged in the design and proposed conduct through the Community Advisory Board (CAB) prior to recruitment. The $\mathrm{CAB}$ is group of community representatives consisting of religious and local leaders, and various community representatives, including adolescents. $\mathrm{CAB}$ meetings will be held monthly during study implementation.

\section{Ethical and dissemination}

This study has been approved by the University of the Witwatersrand's Human Research Ethics Committee (approval number: M200328), the Malawi's NHSRC (approval number: 19/10/2412) and the University of North Carolina at Chapel Hill's Institutional Review Board (approval number: 19-2559). Written informed consent (online supplemental file 1) will be obtained from all participants. Research data will be de-identified and kept under a double-lock system and in password protect databases on the University of North Carolina Project Malawi server. This study has been registered with the US National Library of Medicine at the National Institutes of Health Clinical Trial Registry on 18 December 2020. Participants shall report any unintended harm subjected by the study to the NHSRC. Results will be disseminated via publication in a peer-reviewed journal and presentations at relevant scientific conferences and meetings.

\section{DISCUSSION}

To end the AIDS epidemic by 2030, the UNAIDS recommended use of combination HIV prevention approaches. ${ }^{1}$ VMMC is the only key HIV prevention strategy solely for men. Malawi continues to struggle to achieve its VMMC national target to circumcise $80 \%$ of men aged 10-49 years in priority districts by 2025 , necessitating the need for effective implementation strategies to scale-up uptake of VMMC. As shown earlier, barriers to uptake of VMMC in Malawi include religious and cultural influences; fear of infection, bleeding and pain; limited access to VMMC services; costs associated with accessing VMMC; lack of belief that circumcision can reduce the risk of HIV acquisition and lack of knowledge about the health benefits of VMMC. ${ }^{6-11}$ To address some of these barriers, we designed an interventional implementation study that will evaluate the effectiveness, acceptability, appropriateness and feasibility of intensified health education, SMS/telephonic reminder and transport reimbursement as strategies for scale up of uptake of VMMC among men with STIs in Malawi.

Intensified health education was selected to address barriers resulting from lack of knowledge on VMMC such as insufficient health benefits, lack of belief that circumcision can reduce the risk of HIV acquisition, fear of infection, bleeding and pain, and religious and cultural influences. Health education provided through one-on-one counselling, a video clip and health promotion booklets improved knowledge and perceptions of VMMC and resulted into increased uptake of VMMC within 4 months among men with STIs in China. ${ }^{31}$ In another study, adolescent-targeted one-on-one comprehensive education improved understanding of the benefits of VMMC and increased uptake of VMMC among adolescents in Tanzania. ${ }^{30}$ In our study, we intend to use a combination of comprehensive group education and one-on-one education potentially making this approach more effective and acceptable. As part of the education, we intend to use a female champion. Female partners play an important role in men's decision to uptake VMMC. ${ }^{38-40}$ We expect that using a female champion will make VMMC appealing to other females (partners/ peers) who may influence their male partners to receive circumcision. We anticipate that intensified health education will be effective, acceptable, appropriate and feasible.

To ensure adequate access to VMMC services, we will link men to the nearest public facility providing VMMC services and remind men of their appointments through SMS/telephonic tracing. SMS/telephonic tracing/ reminders have been used to improve many health outcomes. Systematic reviews on SMS and telephonic tracing consistently report improved numerous clinical outcomes across many settings, including in low-income and middle-income countries. ${ }^{32} 4142$ SMS/telephonic reminders have been effectively used to remind men of their VMMC appointments, ${ }^{15}$ and postoperative visits after VMMC. ${ }^{43}$ They are highly usable and acceptable, ${ }^{33}$ and cost effective. ${ }^{44}$ We expect that SMS/telephonic tracing will be effective, acceptable, appropriate and feasible in reminding men with STIs of their VMMC appointment. However, its effect in this study may likely be slightly diminished if some men do not have access to a mobile phone since about $48 \%$ of the Malawian population had mobile phone access by $2019 .{ }^{45}$

In addition, other structural barriers such as costs associated with accessing VMMC services hinder men from accessing VMMC services. ${ }^{43}$ To partly minimise this burden, our study will provide transport reimbursements to men who will receive medical circumcision. Economic compensations of any form, such as food/transport 
vouchers or conditional cash transfers, have effectively increased uptake of VMMC and are well accepted. ${ }^{34} 35$ Though economic compensations have been shown to be favourable in cost, cost-effectiveness analysis is still lacking. ${ }^{46}$ We expect that transport reimbursement will be effective, acceptable and appropriate in our study. However, feasibility in real-world settings may be a challenge. Regardless, we believe that results from our study will inform policy-makers on the effectiveness of economic compensation on uptake of VMMC among men with STIs at high risk of HIV.

As a potential strength, this study will evaluate different combinations of strategies of the VMMC demand creation intervention. We anticipate that the combined effect of the strategies into one intervention will have a greater impact on the uptake of VMMC among men with STIs. In addition to measuring the impact of the intervention, implementation outcomes, acceptability, appropriateness and feasibility of the different combination of strategies will be assessed using mixed methods. Assessing these implementation outcomes will provide a better understanding of the outcome of the intervention (what worked and why it worked). The pre-intervention and post-intervention quasi-experimental study design used in this study has limited strength of evidence; however, we believe this design has been strengthened by adding a prospective observational study design and the concurrent exploratory mixed methods approach evaluation of the intervention. Given the available resources, timeline of the study and the set up of the study site, we could not randomly allocate the intervention strategies to individuals and/or clusters. However, the pragmatic design proposed in this study will allow us to evaluate the impact of the intervention in a 'real-world' setting, likely making our results more generalisable. A cluster randomised study would have required a lot of resources and time to execute, while a randomised controlled trial of individuals would have been difficult to execute without a high likelihood of contamination of the intervention strategies between study groups given the study setting. In addition, the study will follow men for 30 days only from the time they are offered circumcision. Men who will undergo circumcision after 30 days will not be captured by the study, which may result in an underestimation of the impact of the study. Nonetheless, we expect this study to provide insight into the best strategies to use to increase the uptake of VMMC that can be scaled up to other settings.

\footnotetext{
Author affiliations

${ }^{1}$ University of North Carolina Project, Lilongwe, Malawi

${ }^{2}$ Department of Epidemiology and Biostatistics, University of the Witwatersrand, Johannesburg, South Africa

${ }^{3}$ Department of Medicine, Institution for Global Health and Infectious Diseases, University of North Carolina School of Medicine, Chapel Hill, North Carolina, USA

${ }^{4}$ Health and Society Division, School of Public Health, University of the

Witwatersrand, Johannesburg, South Africa

${ }^{5}$ Implementation Science Unit, Right to Care, Centurion, South Africa
}

Twitter Mitch M Matoga @UNCMalawi and Sara Jewett @snieuwoudt

Acknowledgements We would like to thank the Malawi Ministry of Health Department of HIV/AIDS, the Lilongwe District Health Office and Bwaila STI clinic team. We would like to thank Martin Kapito, the VMMC program officer at the Department of HIV/AIDS, Malawi Ministry of Health.

Contributors MMM, MCH, SN and CC contributed to the conceptualisation and design of the study. MMM drafted this manuscript. MCH, SN and CC reviewed and approved the manuscript.

Funding This project is being supported by NIH Fogarty (grant numbers: D43 TW009340, D43 TW010060 and D43 TW009774-06). The study sponsors have not had any role in the study design and will not play a role in collection, management, analysis and interpretation of data; writing the report; and the decision to submit the report for publication.

Competing interests None declared.

Patient consent for publication Not applicable.

Provenance and peer review Not commissioned; externally peer reviewed.

Supplemental material This content has been supplied by the author(s). It has not been vetted by BMJ Publishing Group Limited (BMJ) and may not have been peer-reviewed. Any opinions or recommendations discussed are solely those of the author(s) and are not endorsed by BMJ. BMJ disclaims all liability and responsibility arising from any reliance placed on the content. Where the content includes any translated material, BMJ does not warrant the accuracy and reliability of the translations (including but not limited to local regulations, clinical guidelines, terminology, drug names and drug dosages), and is not responsible for any error and/or omissions arising from translation and adaptation or otherwise.

Open access This is an open access article distributed in accordance with the Creative Commons Attribution Non Commercial (CC BY-NC 4.0) license, which permits others to distribute, remix, adapt, build upon this work non-commercially, and license their derivative works on different terms, provided the original work is properly cited, appropriate credit is given, any changes made indicated, and the use is non-commercial. See: http://creativecommons.org/licenses/by-nc/4.0/.

\section{ORCID iDs}

Mitch M Matoga http://orcid.org/0000-0002-6542-8193

Sara Jewett http://orcid.org/0000-0002-6658-2061

\section{REFERENCES}

1 UNAIDS. On the fast-track to end AIDS. 2016-2021 strategy. Geneva, Switzerland: UNAIDS and WHO, 2021.

2 UNAIDS. Voluntary medical male circumcision: remarkable progress in the scale-up of volontary medical male circumcision as a HIV prevention intervention in 15 eastern and Southern African Countries. Geneva, Switzerland: UNAIDS and WHO, 2019.

3 UNAIDS \& World Health Organisation (WHO). Voluntary medical male circumcision: progress report. Geneva, Switzerland, 2021.

4 National AIDS Commission. National VMMC communication strategy 2020-2025. Lilongwe, Malawi: National AIDS Commission, 2019.

5 Malawi Ministry of Health. Malawi voluntary medical male circumcision strategy and national scale-up plan. Lilongwe, Malawi: Malawi Ministry of Health, 2020.

6 Umar E, Mandalazi P, Jere D, et al. Should female health providers be involved in medical male circumcision? Narratives of newly circumcised men in Malawi. Malawi Med J 2013;25:72-7.

7 Ngalande RC, Levy J, Kapondo CPN, et al. Acceptability of male circumcision for prevention of HIV infection in Malawi. AIDS Behav 2006;10:377-85.

8 Masese RJ, Chimango JL, Mbirimtengerenji ND. Overcoming barriers to uptake of voluntary medical male circumcision in a traditionally Circumcising community in Machinga District, Malawi. World J AIDS 2017;07:40-58.

9 Shacham E, Godlonton S, Thornton RL. Perceptions of male circumcision among married couples in Rural Malawi. J Int Assoc Provid AIDS Care 2014;13:443-9.

10 Rennie S, Perry B, Corneli A, et al. Perceptions of voluntary medical male circumcision among circumcising and non-circumcising communities in Malawi. Glob Public Health 2015;10:679-91.

11 Chilimampunga C, Lijenje S, Sherman J, et al. Acceptability and feasibility of early infant male circumcision for HIV prevention in Malawi. PLoS One 2017;12:e0175873.

12 National AIDS Commission. Malawi National Strategic Plan for HIV and AIDS. Lilon, Malawi: National AIDS Commission, 2020. 
13 Avert. Voluntary medical male circumcision for HIV prevention. Available: https://www.avert.org/professionals/hiv-programming/ prevention/voluntary-medical-male-circumcision [Accessed 26 May 2021].

14 Awad SF, Sgaier SK, Tambatamba BC, et al. Investigating voluntary medical male circumcision program efficiency gains through subpopulation prioritization: insights from application to Zambia. PLoS One 2015;10:e0145729.

15 Msungama W, Menego G, Shaba F, et al. Sexually transmitted infections (STI) and antenatal care (ANC) clinics in Malawi: effective platforms for improving engagement of men at high HIV risk with voluntary medical male circumcision services. Sex Transm Infect 2021;97:345-50.

16 Morris BJ, Hankins CA. Effect of male circumcision on risk of sexually transmitted infections and cervical cancer in women. Lancet Glob Health 2017;5:e1054-5.

17 Dickson KE, Tran NT, Samuelson JL, et al. Voluntary medical male circumcision: a framework analysis of policy and program implementation in eastern and southern Africa. PLoS Med;8:e1001133.

18 Auvert B, Taljaard D, Lagarde E, et al. Randomized, controlled intervention trial of male circumcision for reduction of HIV infection risk: the ANRS 1265 trial. PLoS Med 2005;2:e298-1122.

19 Gray RH, Kigozi G, Serwadda D, et al. Male circumcision for HIV prevention in men in Rakai, Uganda: a randomised trial. Lancet 2007;369:657-66.

20 Matoga M, Hosseinipour MC, Jewett S, et al. Effects of HIV voluntary medical male circumcision programs on sexually transmitted infections. Curr Opin Infect Dis 2021;34:50-5.

21 National Statistical Office. Malawi demographic and health survey 2015-16, 2015

22 Malawi Ministry of Health. Integrated HIV program report April - June 2018. Lilongwe, Malawi, 2018

23 Malawi Ministry of Health. Malawi population-based HIV impact assessment (MPHIA) 2015-2016 report. Lilongwe, Malawi: Ministry of Health, 2018.

24 Malawi Ministry of Health. Integrated HIV program report October December 2020. Lilongwe, Malawi: Ministry of Health, 2021.

25 Chen JS, Matoga M, Pence BW, et al. A randomized controlled trial evaluating combination detection of HIV in Malawian sexually transmitted infections clinics. J Int AIDS Soc 2021;24:1-9.

26 Powell BJ, Waltz TJ, Chinman MJ, et al. A refined compilation of implementation strategies: results from the expert recommendations for implementing change (ERIC) project. Implement Sci 2015;10:21.

27 Sgaier SK, Baer J, Rutz DC, et al. Toward a systematic approach to generating demand for voluntary medical male circumcision: insights and results from field studies. Glob Health Sci Pract 2015;3:209-29.

28 Maibvise C, Mavundla TR. A model to promote the uptake of male circumcision as an HIV-preventive measure in high HIV and low male circumcision prevalence settings. Health SA Gesondheid 2019;24.

29 Proctor EK, Powell BJ, McMillen JC. Implementation strategies: recommendations for specifying and reporting. Implement Sci 2013;8:1-11.

30 Boyee D, Peacock E, Plotkin M, et al. What messages are adolescent voluntary medical male circumcision (VMMC) clients getting and how? findings from an observational study in Tanzania. AIDS Behav 2017;21:1383-93.
31 Wang Z, Feng T, Lau JTF. Needs assessment and Theory-Based promotion of voluntary medical male circumcision (VMMC) among male sexually transmitted diseases patients (MSTDP) in China. AIDS Behav 2016;20:2489-502.

32 Schwebel FJ, Larimer ME. Using text message reminders in health care services: a narrative literature review. Internet Interv 2018;13:82-104

33 Feldacker C, Holeman I, Murenje V, et al. Usability and acceptability of a two-way texting intervention for post-operative follow-up for voluntary medical male circumcision in Zimbabwe. PLoS One 2020;15:e0233234.

34 Carrasco MA, Grund JM, Davis SM, et al. Systematic review of the effect of economic compensation and incentives on uptake of voluntary medical male circumcision among men in sub-Saharan Africa. AIDS Care 2018:30:1071-82.

35 Kennedy CE, Yeh PT, Atkins K, et al. Economic compensation interventions to increase uptake of voluntary medical male circumcision for HIV prevention: a systematic review and metaanalysis. PLoS One 2020;15:e0227623.

36 Proctor E, Silmere H, Raghavan R, et al. Outcomes for implementation research: conceptual distinctions, measurement challenges, and research agenda. Adm Policy Ment Health 2011;38:65-76.

37 Weiner BJ, Lewis CC, Stanick C, et al. Psychometric assessment of three newly developed implementation outcome measures. Implement Sci 2017:12:108.

38 Lanham M, L'Engle KL, Loolpapit M, et al. Women's roles in voluntary medical male circumcision in Nyanza Province, Kenya. PLoS One 2012;7:44825.

39 Cook R, Jones D, Redding CA, et al. Female partner acceptance as a predictor of men's readiness to undergo voluntary medical male circumcision in Zambia: the spear and shield project. AIDS Behav 2016;20:2503-13.

40 Wambura M, Mahler H, Grund JM, et al. Increasing voluntary medical male circumcision uptake among adult men in Tanzania. AIDS 2017;31:1025-34.

41 Lee SH, Nurmatov UB, Nwaru BI, et al. Effectiveness of mHealth interventions for maternal, newborn and child health in low- and middle-income countries: systematic review and meta-analysis. $J$ Glob Health 2016;6:010401.

42 Kannisto KA, Koivunen MH, Välimäki MA. Use of mobile phone text message reminders in health care services: a narrative literature review. J Med Internet Res 2014;16:e222.

43 Odeny TA, Bailey RC, Bukusi EA, et al. Text messaging to improve attendance at post-operative clinic visits after adult male circumcision for HIV prevention: a randomized controlled trial. PLOS One 2012; 7:e43832.

44 Babigumira JB, Barnhart S, Mendelsohn JM, et al. Costeffectiveness analysis of two-way texting for post-operative followup in Zimbabwe's voluntary medical male circumcision program. PLoS One 2020;15:e0239915.

45 The World Bank. Mobile cellular subscriptions (per 100 people) Malawi | Data. Available: https://data.worldbank.org/indicator/IT.CEL. SETS.P2?locations=MW [Accessed 1 Sep 2021]

46 Thirumurthy $\mathrm{H}$, Masters SH, Rao S, et al. Effect of providing conditional economic compensation on uptake of voluntary medical male circumcision in Kenya: a randomized clinical trial. JAMA 2014;312:703-11. 